\title{
Postgraduate Course ERS Glasgow 2004 Surgical approach to multiresistant cavitary mycobacteriosis
}

\section{Educational aims}

I To explain the present importance of surgery in TB management.

1 To describe the indications for surgery.

1 To discuss the different stages of surgery, along with important points to consider.

1 To present examples of data from one institution to help demonstrate the issues to be considered in surgical management.

\section{Summary}

Surgery remains an important adjunctive method in the treatment of MDR-TB. In this article, adapted from the European Respiratory Society (ERS) School Course on "Multi-resistant tuberculosis and atypical mycobacterial disease", held at the 2004 ERS congress in Glasgow, the indications for surgical management in patients with MDR-TB are discussed, together with data from 75 patients with MDR-TB operated on in the Sureyyapasa Thoracic and Cardiovascular Diseases Teaching Hospital, Turkey, between 1993 and 2003.

In most parts of the world, the use of thoracic surgery has stemmed from the management of inflammatory lung diseases. Some 50 years ago, many outstanding journals, which today publish modern cardiac or thoracic papers, were full of studies related to surgery for tuberculosis (TB): for example, thoracoplasty, decortication or pulmonary resection. Fortunately, effective anti-TB drugs emerged in the 1950s and 1960s, and, hence, the role of thoracic surgeons was limited to the management of its complications, i.e. destroyed lung, empyema, bronchostenosis, bronchopleural fistula, pachypleuritis and haemoptysis. However, the anti-TB drugs were antibiotics, and, over time, Mycobacterium tuberculosis has changed its structure in response to the antibiotics and resistance to the treatment of TB has emerged.

There are other factors that have contributed to the development of resistance, such as increasing prevalence of immunocompromising diseases, inappropriate therapies, inadequate monitoring and poor patient compliance. However, the end result is that surgery must take up its historical role once again to help in solving the problem as an adjunctive modality. Infection with multi-drug resistant (MDR)-TB is the most common indication for surgery in patients with TB today.
S. Halezeroglu
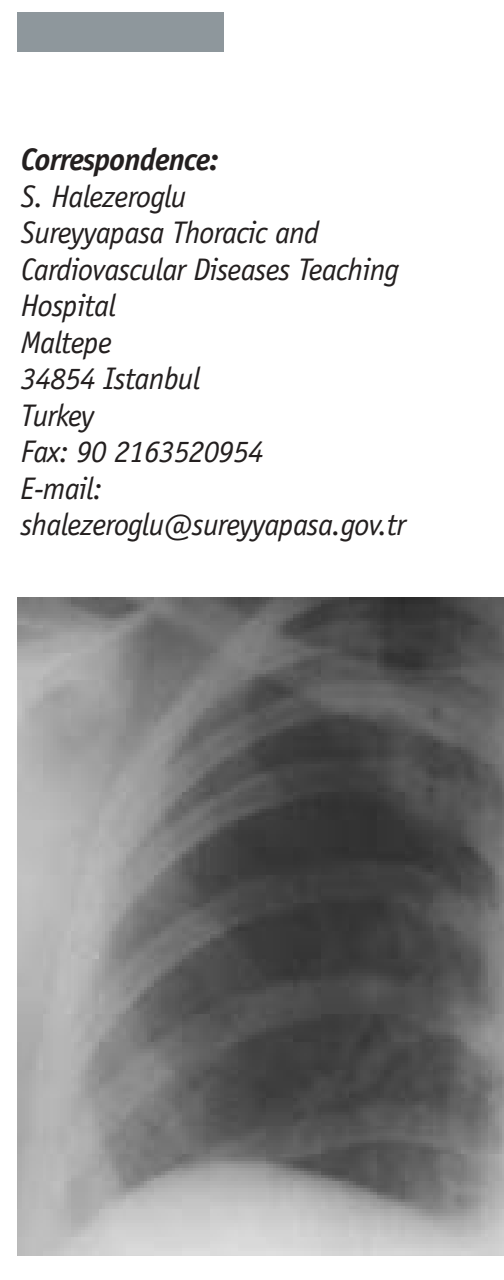

CrossMark \& click for updates 

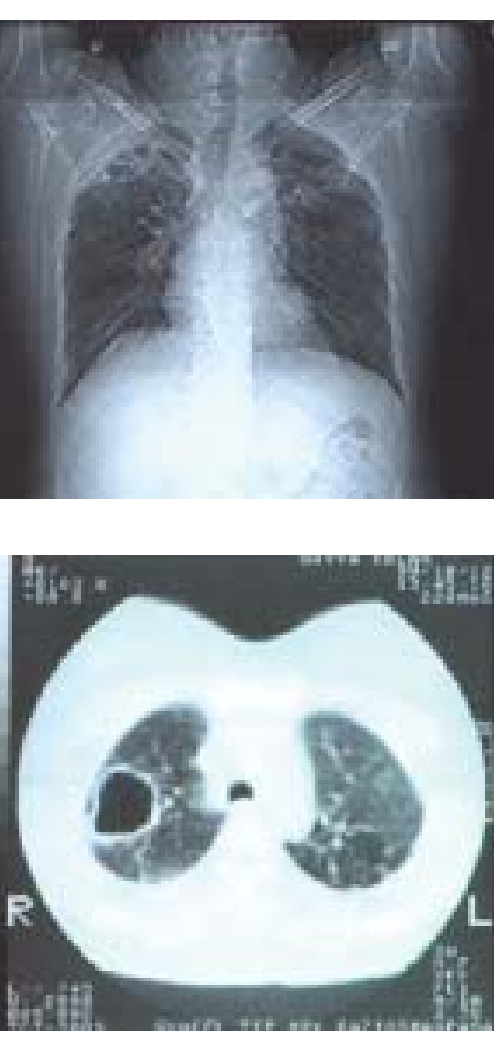

\section{Indications for surgery in MDR-TB}

Pulmonary resection should be undertaken in the setting of localised disease, persistent sputum positivity or patient intolerance of medical therapy. It must be emphasised that all types of patients with TB must be first treated medically by pneumologists. Teamwork is of paramount importance in the management of MDR-TB: the better the cooperation between the thoracic surgeon and the chest physician, the better the results of treatment.

Surgery is indicated when pneumologists consider that the patient cannot be cured by medical therapies. It is important to note that at least 3 months of treatment should be given before surgery, if it is feasible at all. The role of the surgeon in this circumstance is to look for indications for surgery and to decide if the patient is a suitable candidate for resectional surgery. Surgery is performed mainly to remove the heavy bacterial burden within the cavity. A destroyed lobe or lung acts in a similar way to cavity disease, and should also be removed when treatment fails.

There are four main indications for surgery, all of which presume that there is adequate cardiopulmonary reserve, as follows:

$>$ Failure to convert (failure of anti-TB drug regimen to convert sputum from positive smear to negative smear): positive sputum smear or culture within 2 months before operation, despite at least 4-6 months of treatment with an adequate drug regimen; presence of destroyed lobe or lung (with cavitation, bronchiectasis and bronchostenosis) is associated with high risk of treatment failure or relapse.

$>$ Previous relapse(s): previous TB history of two or more relapses and/or one or more relapses during MDR-TB treatment course; if relapse did occur, it may be difficult to re-establish sputum culture conversion.

$>$ High profile of drug resistance: infection with strains of $M$. tuberculosis resistant to four or more drugs.

$>$ High or potential risk of relapse: patients not falling into any of the previous categories, but considered likely to relapse despite conversion, as gauged by the presence of a destroyed lung or a lobe.

When the probability of negative bacteriological conversion of sputum seems high (i.e. less destroyed lung, initial resistance to isoniazid
(INH) and rifampicin (RFP), only resistant to INH and RFP), chemotherapy should be continued for an additional 2-3 months. Should any significant improvement occur clinically then chemotherapy should continue; while "no change" or worsening in clinical, radiological or bacteriological findings indicates surgery.

\section{Surgical resection of lung disease}

\section{Pre-operative period}

The decision to perform surgery and the extent of surgery, i.e. lobectomy or pneumonectomy, should be made after anatomical localisation of the disease by computed tomography (CT) scan. Perfusion scans are useful in establishing how much functioning lung is likely to be removed. Bronchoscopy is necessary to rule out endobronchial TB, which is quite a rare but important condition that increases post-operative complication rates. Basic spirometry (i.e. forced expiratory volume in one second and forced vital capacity) is adequate for assessing lung function in the majority of patients. Electrocardiography is useful for excluding pulmonary hypertension, which would contraindicate surgery.

In a patient who has not undergone sputum culture conversion, surgery should only be performed when there is no further possibility of an adequate chemotherapeutic regimen. Positive sputum for M. tuberculosis and surgery is not a desirable association. However, sputum negativity by drugs cannot always be achieved; surgery remains the only tool to achieve this goal (to convert sputum from positive culture to negative culture).

Most, if not all, of the patients considered for surgery are in poor physical condition. Serum albumin levels are generally low and must be increased during the pre-operative period. As blood loss from the adhesions between the lung and the chest wall, pericardium and diaphragm is not unusual during separation of the lung, at least four units of blood must be prepared before the operation.

\section{Surgery}

During surgery, a double-lumen endotracheal tube is used to isolate the lungs. Prophylaxis with parenteral antibiotics must be started just prior to the incision. Posterolateral thoracotomy is performed and muscles are saved for potential use in the management of possible post-operative 
bronchopleural fistula (BPF) complication. Lobar resection is the goal of surgery. Pneumonectomy, especially right pneumonectomy, should be avoided whenever possible. There is only limited opportunity for segmental/limited resection.

Generally, the disease will be located in one lobe, with some nodular disease in another lobe or lobes. Nodules in the lungs also contain bacilli, but not as much as in a cavity or a destroyed lobe. Any attempt to remove all the nodules by resecting the entire lobe or by limited resection only causes increased post-operative difficulties and is unnecessary.

If the disease does not invade the entire lung, it locates predominately in the right upper lobe. In patients with MDR-TB, destruction of the left lung requiring pneumonectomy occurs more frequently than in the right lung. Although right-sided total lung destruction is rare, the removal of only the right upper lobe is not always possible because of invasion into the superior segment of the lower lobe with TB. Frequently, there is no plane between the upper lobe and superior segment of the lower lobe. The only way to remove the upper lobe is to separate the lobes by sharp dissection using electrocautery. Linear stapling devices are not generally useful because of thickened parenchyma in association with the disease. In this situation, the surgeon must decide whether to: 1) remove only the upper lobe and leave the lower lobe (that cannot be expanded) open to complication (i.e. prolonged air leak, empyema); or 2) remove all three lobes.

Pre-operative perfusion scan is one tool that can help with the decision. However, the intraoperative finding of an experienced surgeon is the most important consideration in this situation. The possibility of complications if the partially diseased lower lobe is left unresected (because of nodular disease and/or parenchymal air leakage from the separated superior segment of the lower lobe) must be well above the complication rate of a right pneumonectomy if the entire right lung is to be resected. If the probability of post-operative complications does not outweigh the complications of right pneumonectomy, then only the upper lobe should be resected.

Although pneumonectomy is not a desirable resection, complete lung destruction mandates total removal of the lung. Preservation of the bronchial stump by very careful dissection and by buttressing with adjacent structures may decrease the BPF. If the risk of infection in the postpneumonectomy cavity is high, irrigation of the cavity with saline, which maintains mechanical cleaning, might be helpful in addition to parenteral antibiotics.

The resected specimen should be sent for histology, culture and susceptibility. The patient should then be redirected to the related pneumology unit to arrange drug regimen and follow-up. Even when the entire diseased lung is resected, anti-TB treatment should continue for 18-24 months after surgery.

\section{Post-operative period: complications}

The most important complication of surgery for TB is BPF. This is more common after right pneumonectomy when compared with all other resections. BPF rate is closely related to positive sputum before surgery, lower albumin level and presence of pre-operative empyema. BPF occurring after TB surgery cannot be managed in the same way as in patients who undergo pneumonectomy for lung cancer. Although muscle flap appears to be a good method to close the stump, patients are generally weak and only have thin muscles. Transsternal-transpericardial closure of the stump must be very carefully used, since the infection can be disseminated to the vital organs in the mediastinum. Re-closure of the stump by thoracotomy in such a diseased bronchus is very hard to achieve. It is our opinion that thoracostoma (open-window thoracostomy) or thoracoplasty is the best way to save the lives in BPF patients with MDR-TB. Other complications of surgery include bleeding, atelectasis, prolonged air leak, empyema, post-pneumonectomy pulmonary oedema and Horner's syndrome.

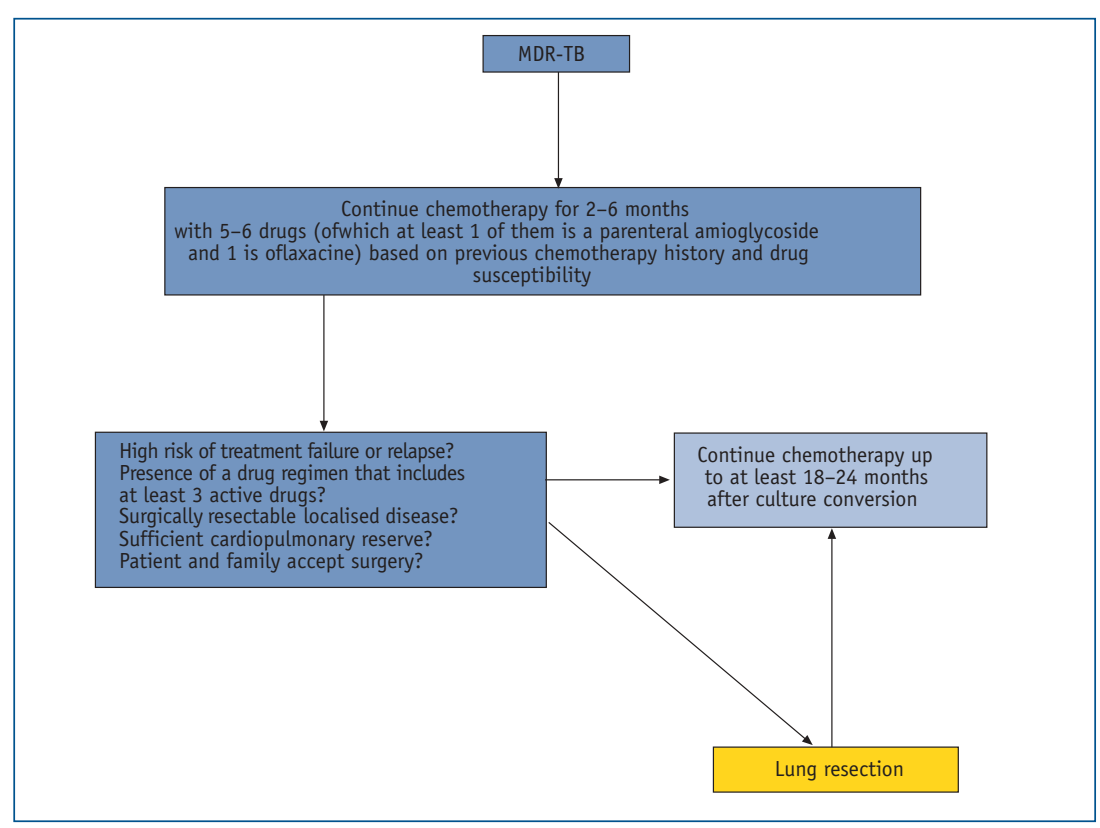




\section{Suggested further reading}

Pomerantz M, Mault JR. History of resectional surgery for tuberculosis and other mycobacterial infections. Chest Surg Clin N Am 2000; 10: 131-133, ix. All medical staff interested in the treatment of TB should read this unique paper. Every thoracic surgeon must read it before proceeding with surgery for TB.

Iseman MD, Madsen L, Goble M, Pomerantz M. Surgical intervention in the treatment of pulmonary disease caused by drug-resistant Mycobacterium tuberculosis. Am Rev Respir Dis 1990; 141: 623-625.

One of the first reports on the subject, which defines the indications and results of surgery in MDR-TB in 1990.

Iseman MD. Treatment of multidrug-resistant tuberculosis. N Engl J Med 1993; 329: 784-791.

In 1993, Iseman emphasised the importance of dissemination of MDR-TB patients. He highlights the urgent need for local programmes for prevention, control and research in order to identify new medications and systems for their delivery.

Tahaoglu K, Torun T, Sevim T, et al. The treatment of multidrugresistant tuberculosis in Turkey. N Engl J Med 2001; 345: 170-174.

This paper documents the treatment strategy in MDR-TB at Sureyyapasa Hospital in Turkey. The results of chemotherapy, as well as the surgery against the disease are explained.

van Leuven M, De Groot M, Shean KP, von Oppell UO, Willcox $P A$. Pulmonary resection as an adjunct in the treatment of multiple drug-resistant tuberculosis. Ann Thorac Surg 1997; 63: 1368-1372,

discussion 1372-1373.

The indications for surgery in MDR-TB are clearly defined. The safety of surgery in experienced units are emphasised.

\section{Results}

Between 1993 and 2003, 77 surgical interventions were performed at Sureyyapasa Thoracic and Cardiovascular Diseases Teaching Hospital in 75 patients with MDR-TB. The hospital has a comprehensive TB unit, which was established in 1993 to supervise the treatment and follow-up of patients with MDR-TB. The unit offers long-term hospitalisation with all the required drug therapy. All expenses during treatment (hospitalisation, drugs and surgery) are covered by the state organisation and, therefore, patients do not incur any

\section{Table 1 Characteristics of patients}

\begin{tabular}{|lc|}
\hline Patients & 75 \\
Operations & $77^{\sharp}$ \\
Sex & \\
$\quad$ Male & 60 \\
$\quad$ Female & 15 \\
Mean age & 38 years \\
Laterality & \\
$\quad$ Unilateral & $46(61 \%)$ \\
$\quad$ Bilateral & $29(39 \%)$ \\
$\quad$ Left & $41(55 \%)$ \\
$\quad$ Right & $34(45 \%)$ \\
Sputum (before operation) & \\
$\quad$ Positive & $7(9 \%)$ \\
$\quad$ Negative & $68(91 \%)$ \\
Time between re-treatment & \\
and surgery & $3(2-6)$ months \\
\hline : two patients underwent two re-thoracotomies for pulmonary \\
resection for MDR-TB.
\end{tabular}

\section{Table 2 Operations performed}

\begin{tabular}{|lcc|}
\hline Operations & Left & Right \\
Pneumonectomy & 27 & 11 \\
Completion pneumonectomy" & 2 & 2 \\
Upper lobectomy & 9 & 12 \\
Lower lobectomy & 1 & 4 \\
Upper + lower lobe & 2 & 4 \\
supplementary segmentectomy & & \\
Upper + middle lobectomies & & 2 \\
Lingulectomy & 1 & \\
Total & 42 & 35 \\
\hline ": three out of four patients had undergone lobectomy for other \\
reasons ( $n=2$ ) and TB ( $n=1$ ) before. One patient had undergone \\
lobectomy for MDR-TB in this series and then underwent \\
completion pneumonectomy.
\end{tabular}

expenses. Thoracic Surgery and Pneumology departments work in very close cooperation.

The following tables summarise the results of surgery performed in 75 patients with MDR-TB.

Table 3 Post-operative complications

\begin{tabular}{|lll|}
\hline BPF & 4 & $(5.1 \%)$ \\
Bleeding & 5 & $(6.4 \%)$ \\
Prolonged air leak & 5 & $(6.4 \%)$ \\
Empyema & 4 & $(5.1 \%)$ \\
Atelectasis & 2 & $(2.6 \%)$ \\
Horner's syndrome & 2 & $(2.6 \%)$ \\
Delayed re-expansion & 1 & $(1.3 \%)$ \\
Total morbidity & 23 & $(29.9 \%)$ \\
Mortality & 2 & $(2.6 \%)$ \\
\hline
\end{tabular}

Table 4 Long-term results after surgical resection plus chemotherapy for MDR-TB

\begin{tabular}{|lll|}
\hline Cure $^{\sharp}$ & 61 & $(81.3 \%)$ \\
Probable cure & 4 & $(5.3 \%)$ \\
Treatment failure & 1 & $(1.3 \%)$ \\
Relapse (1 dead) & 2 & $(2.6 \%)$ \\
Operative deaths & 2 & $(2.6 \%)$ \\
Incomplete drug regimen & 1 & $(1.3 \%)$ \\
Lost to follow-up & 4 & $(5.3 \%)$ \\
\hline ": negative smears and cultures throughout treatment for $\geq 18$ \\
months; ${ }^{q}$ : negative smears and cultures throughout treatment for \\
$\geq 6$ months.
\end{tabular}

\section{Conclusion}

Surgery remains an important adjunctive tool in the management of patients with MDR-TB. The success of surgery in MDR-TB depends on the following criteria:

$>$ cooperation and teamwork (chest physicians and thoracic surgeons);

$>$ patient compliance to complete the drug regimen, which lasts 18-24 months postoperatively;

$>$ familiarity of surgeon with the surgery for TB disease;

$>$ adherence to pre-operative and post-operative precautions;

$>$ careful follow-up by chest physicians. 


\section{Educational questions}

1. What is the main goal of surgery for MDR-TB?
a) To remove the entire diseased lung.
b) To remove the heavy bacterial burden within the cavity.
c) To prevent long-term drug use.
d) To convert the sputum culture positivity.

2. Which of the following is not an indication for surgery in MDR-TB?
a) Positive sputum after 1 month of re-treatment for MDR-TB.
b) Previous TB history of one or more relapses during MDR-TB treatment course.
c) High profile of drug resistance.
d) High or potential risk of relapse.

3. Which of the following patients with MDR-TB has the most favourable post-operative outcome?
a) Patient with positive sputum for $M$. tuberculosis.
b) Patient with right-sided destroyed lung.
c) Patient with serum albumin level of $4.5 \mathrm{~g} \cdot \mathrm{dL}^{-1}$ and left lower lobe disease.
d) Patient who has history of relapse due to his/her inappropriate drug intake.
e) Patient with dense cavity in the right upper lobe with nodularity in the lower lobe.

4. What should the surgeon do after surgery?
a) Discharge the patient without further medication if all the diseased lung was removed.
b) Discharge the patient with medication against MDR-TB and invite him/her to control at the surgical unit during the treatment course.
c) Redirect the patient to the pneumology unit to arrange medical therapy and follow-up.
d) The next step is closely related to the bacteriological and pathological report from the resected specimen.

5. Which of the following is an unnecessary diagnostic tool before the operation?
a) MRI of the lung.
b) $\mathrm{CT}$ of the lung.
c) Sputum culture.
d) Bronchoscopy.
e) Perfusion scan, if the removal of the entire lung is a possibility.

6. Which of the following is the least important in the success of surgery?
a) Good patient selection in regard to age and sex.
b) Patient compliance.
c) Follow-up of the patient for at least 2 years.
d) Experience of the surgeon with TB surgery.
e) Teamwork between surgery, pneumology and microbiology units.

7. Which of the following is the most important part of the team in the surgical management of MDR$\mathrm{TB}$ ?
a) Pneumologist.
b) Microbiologist.
c) Thoracic surgeon.
d) All are equally important.

\section{The correct answers are:}
$1-b$
2- a
3- $c$
4- $c$
5- a
6- a
7-d 